\title{
Improving data management practices in the Portuguese HIV/AIDS surveillance system during a time of public sector austerity
}

\author{
Tara Shivaji, Helena Cortes Martins \\ Directorate General of Health and the National Institute of Health Dr Ricardo Jorge, Lisbon, Portugal, European Programme for Intervention \\ Epidemiology Training (EPIET), European Centre for Disease Prevention and Control (ECDC), Stockholm, Sweden
}

\begin{abstract}
In a climate of public sector austerity, the demand for accurate information about disease epidemiology rises as health program managers try to align spending to health needs. A policy of case re-notification to improve HIV information quality resulted in a nine-fold increase in the number of case reports received in 2013 by the Portuguese HIV surveillance office.
\end{abstract}

We used value stream mapping to introduce improvements to data processing practices, identify and reduce waste. Two cycles of improvement were trialled. Before intervention, processing time was nine minutes and 28 seconds (95\% $\mathrm{Cl} 8: 53-10: 58)$ per report. Two months post intervention, it was six minutes and 34 seconds (95\% $\mathrm{Cl}$ 6:25-6:43). One year after the start of the project, processing time was five minutes and 20 seconds (95\% Cl 1:46-8:52).

\section{Problem}

The aim of this project was to reduce the time spent processing HIV surveillance data from paper into electronic information. Notification of HIV has been a statutory requirement in Portugal since 2005. Individual paper case reports are sent to the national surveillance office and processed by a team of two administrative staff before being transcribed into the national database. An evaluation of the surveillance system found long delays, the average time between diagnosis and notification was two years.[1]

The recent European sovereign debt crisis resulted in widespread public sector austerity in Portugal. Pushed to justify the allocation of resources according to need, the demand for high quality HIV epidemiology information in Portugal increased. To improve the quality of HIV information, all existing HIV positive patients in Portugal were notified during 2013, irrespective of previous notification. The workload of the national surveillance office increased from approximately 2500 notifications per year to 23,000 notifications.

The ongoing austerity meant no additional resources to assist with processing. Waiting time for HIV information increased and staff felt under increasing pressure to meet international and national reporting deadlines. Space had to be found to securely store the large volume of paper until it could be processed.

\section{Background}

Epidemiologic surveillance is the continuous collection, analysis, interpretation and dissemination of health data to inform public health action.[2] Paper based surveillance systems need to minimise errors as a result of data transcription to ensure the production of good quality information.[3]
HIV information is used to inform prevention and treatment strategies which reduce the mortality, morbidity and limit the health care costs associated with HIV and AIDS.[4] Surveillance is also important for the early detection and control of HIV outbreaks.[5] In some countries, surveillance systems can account for up to $10 \%$ of the total HIV prevention budget [6] and are thus highly vulnerable to cuts in public sector spending and efficiency savings.[7]

\section{Baseline measurement}

The average number of case reports received each day by the national surveillance office in 2012 was six and during 2013 was 96 (range of 0 to 596). Case reports were processed in the order that they were received. We identified the steps that each case report passed through to be processed into HIV information. There were six steps in this process:

1. Receipt of the case reports by mail

2. Writing a soundex code onto the case report

3. Searching the database to identify if the case is a new diagnosis, clinical progression, or duplicate

4. Allocation of a process number

5. Transcription of data

6. Checking the entered data against the paper report for mistakes.

In January 2014, we recorded the times that each step started and stopped for batches of 10 reports. The average time for one report to complete a step was calculated and the time per step summed to calculate the average processing time, which was nine minutes 28 seconds (95\% $\mathrm{Cl} 8: 53-10: 58)$ per report.

In January 2014 by applying processing times to the number of case reports received we calculated a minimum lead time for the production of HIV information of nine months. 


\section{Design}

We used LEAN methodologies to improve processing time. We identified HIV information users; program managers, regional HIV teams, non-governmental organizations, and asked them about their information requirements and priorities. We also reviewed recent ad-hoc requests for HIV information. We produced a process map by following a case report from its receipt to the production of information. We combined the responses of information users and the process map to form a value stream map.

HIV surveillance staff were provided with reading material about LEAN in Portuguese to introduce general concepts. [8] We conducted informal focus groups in the surveillance office ranging from 15 to 30 minutes. Staff were encouraged to use the value stream map to identify wastes and discuss possible improvements. We did not consider improvements that would have required investing in additional equipment or staff.

The Portuguese HIV/AIDS surveillance system uses Epilnfo 7, a free software package for the collection and analysis of disease data developed by the US Centers for Disease Control and Prevention (CDC). We consulted the user manual and online discussion groups for technical help implementing proposed solutions.

\section{Strategy}

We undertook two cycles of change. The first cycle of change occurred between January and March 2014 and focused on reducing wastes.

HIV information users told us that information relating to the most recent years (2010 to 2013) was the highest priority. In response to this, we clearly divided old and recent cases once they were received and prioritised the processing of recent cases in each step.

The consultation with information users revealed that some of the variables collected on the case report form were not used. These questions reflected the Portuguese HIV epidemic of the 1980s and 1990 s and were not relevant to modern program planning. We stopped entering data on these variables to reduce this waste. They also told us that they were interested in new HIV diagnoses and clinical progression to AIDS. In response to this, we stopped entering information about progression from asymptomatic to symptomatic non-AIDS stages.

Using the value stream map we identified that if the database search was not done correctly, duplicate cases could be entered into the database leading to overestimations of the numbers of people living with HIV / AIDS. Furthermore once we did identify a duplicate, processing of other records had to stop whilst the two records were linked. To remove existing duplicates, we used STATA 12 to identify case records with the same soundex code. These records were verified and corrected where necessary. To prevent future duplicates, we introduced a check code. This function of Epilnfo automatically searched for matching soundex codes during data entry and alerted transcribers to the potential duplicate entry of cases.

To prevent spelling and calculation errors we used functions such as drop down menu lists and automatic formula calculation. Variables including age at diagnosis and area of residence could be calculated automatically from entered data entered earlier in the data entry mask.

The second cycle of change took place between March 2014 and December 2014. This cycle of change focused on introducing standard work

When we received case reports with incomplete or missing information, processing would stop as we decided how to handle the case report and whether it could continue to be processed or not. We realised during the discussions that there were some variables which were more frequently missing than others. We developed a series of rules to follow which meant that the process did not have to stop and wait for a decision to be taken.

Comparing the paper based record to a line list of the information entered into the database was laborious and time consuming. No further processing of information occurred whilst this step was taking place. Staff time was spent looking for errors rather than correcting errors or processing. We wrote a series of programs which automatically identified records with errors which could then be corrected.

\section{Results}

At the end of the first cycle (March 2014), we measured the time taken to process batches between 10 to 100 case reports to complete each step and calculated the average time per case report. The sum of these average processing times per report was six minutes and 34 seconds (95\% $\mathrm{Cl}$ 6:25-6:43).

During the second cycle, the number of full time staff reduced from two to one due to maternity leave. The team received 0.8 full time employee administrative support for database searching by the partial redeployment of staff from other laboratory areas. At the end of the second cycle, one year after the project started, the average processing time per case report was measured for a batch of 30 reports and found to be five minutes and 20 seconds $(95 \% \mathrm{Cl}$ 1:46-8:52)

The sustained benefit was seen in data transcribing and the data quality check step and reflects the changes in workload and typing time from automating parts of the data entry mask and reducing over production of information. The average time for database searching increased following staff changes despite the introduction of standard work as staff unfamiliar with the protocols would want to consult with another colleague before making a decision about a case.

In January 2015, the lead time for cases diagnosed between 2010 and 2014 was 20 working days and for cases diagnosed prior to 
2010, lead time was three months.

See supplementary file: ds5369.pptx - "Figure 1; Average processing time per step at baseline and following each cycle of improvement"

\section{Lessons and limitations}

This work set out to improve a process of producing electronic HIV information from paper based case reports. For many countries, paper based systems form the bedrock of communicable disease surveillance and HIV case reporting and surveillance is set to become the standard in describing the epidemiology of the disease as HIV donor and funding priorities change. Outside of communicable disease surveillance, this project has the potential to improve the timeliness of the availability of electronic information, for example updating records in primary care with the discharge letter from a recent hospital admission or outpatient appointment. We found that improving the processing of paper records into electronic information lent itself well to the LEAN methodology. A possible reason for this could be that the processing of paper case reports are more akin to manufacturing and production line processes originally targeted by LEAN. The individual changes that we made within the system are less generalisable and their usefulness to others highly dependent on the operational context.

Staff involvement, senior management support and momentum for change have previously been described as key factors for successful implementation.[9] In our project, engaging front line staff when they were under significant pressure to continue with their current way of working was difficult. Regular feedback of changes in processing time for different steps as well as harnessing and developing their ideas for improvement were particularly important in securing participation.

If we were to design the project again we would systematically record the experiences of staff. Anecdotally, we found that the project had a positive impact on the perceived burden of work and stress they experienced. Staff reported feeling more able to cope with the demands placed on them during and after the project and continued to generate ideas and actively engage with stakeholders after the project.

Not all our changes were feasible in the timescale. We would have liked the soundex code to be printed directly on the case report form which would have allowed us to eliminate the soundex code step. We were not able to implement this change during the period of the project. Our project facilitated a discussion about HIV surveillance to monitor the epidemic in Portugal and what information was required which was currently not collected. The discussion is ongoing and we hope that our change will be implemented once a final decision is reached.

Continued improvement in processing time has been difficult to sustain over the course of one year and highlights the fragility of a service highly dependent on the performance of one or two skilled individuals. Our results show that standardising processes can provide organisational resilience to the effects of staff turnover.

\section{Conclusion}

We set out to improve our data processing practices at a time when the HIV surveillance service experienced real-term cuts in resources, coupled with a nine-fold increase in demand. We achieved a $45 \%$ reduction in processing time following the implementation of LEAN methodology. Using this approach we were able to view the HIV surveillance system in terms of a process. Our actions to eliminate waste were made with an understanding of the purpose and value of the information that we produced. Further work would be valuable to address the reporting delay between diagnosis and notification and thereby improve the overall timeliness of the surveillance system in order to achieve our aim of providing timely and accurate HIV information in Portugal.

\section{References}

1. Mauch S. A situational needs assessment of the Portugese HIV AIDS notification system A Portuguese Experience. Lisbon: The National Coordination of HIV Infection; 2009.

2. German, Robert R., Lee LM. et al. Updated guidelines for evaluating public health surveillance systems. MMWR Recomm Rep 50, no. RR-13 (2001): 1-35.

3. European Centre for Disease Prevention and Control. Data quality monitoring and surveillance system evaluation - A handbook of methods and applications. Stockholm: ECDC; 2014.

4. UNAIDS.Guidelines for Second Generation HIV Surveillance: the next decade. Geneva: Joint United Nations Programme on HIV/AIDS (UNAIDS); 2000.

5. Pharris A, L Wiessing, O Sfetcu, D Hedrich, A Botescu, A Fotiou, GK Nikolopoulos et al. "Human immunodeficiency virus in injecting drug users in Europe following a reported increase of cases in Greece and Romania, 2011." Euro Surveill 16, no. 48 (2011): 14-8.

6. Schwartländer B., Stover J., Walker N. et al. Resource needs for HIV/AIDS. Science 292, no. 5526 (2001): 2434-6.

7. Rechel, Boika, Suhrcke $\mathrm{M}$ et al. Economic crisis and communicable disease control in Europe: a scoping study among national experts. Health Policy 103, no. 2 (2011): 168-75.

8. Suzaki, Kiyoshi. New manufacturing challenge: Techniques for continuous improvement. New York: Simon and Schuster:1987 (Portuguese translation Lean op press 2010).

9. Radnor Z,Walley P. Learning to walk before we try to run: adapting lean for the public sector. Public Money and Management 28, no 1 (2008):13-20.

\section{Declaration of interests}

Nothing to declare.

\section{Acknowledgements}

Dr Antonio Diniz and the National HIV/AIDS Programme, Portugal, 
Dr Marion Muehlen, Dr Paula Vasconcelos, Professor Paulo Nogueira supervisors and coordinators on the European Programme for Intervention Epidemiology Training (EPIET), European Centre for Disease Prevention and Control (ECDC) Stockholm, Sweden

Dr Cameron Stark, Consultant in Public Health, NHS Highland for advice on implementing a LEAN improvement project.

\section{Ethical approval}

The project was exempt from ethical approval as it was a service improvement study which did not directly involve human subjects. 